\title{
Dispositivo para la movilidad autónoma de personas usuarias de sillas de ruedas convencionales
}

\author{
Carlos Montes Rodríguez *, Miguel Herrera Suárez, Edgar Solórzano Guerrero \\ ${ }^{1}$ Universidad Técnica de Manabí. Portoviejo, Ecuador
}

Recibido 4 de abril de 2019. Aceptado 29 de julio de 2019

\begin{abstract}
Resumen - La inclusión social para las personas con discapacidad influye en su desarrollo personal dentro de la sociedad en la cual se encuentran, se disponen de variantes y de ayudas prioritarias para permitir que esta población en situación de discapacidad, pueda desenvolverse de manera autónoma y sin complicaciones en las actividades programas día a día. Al considerar esta problemática el presente trabajo tiene como objetivo desarrollar un dispositivo para el traslado autónomo de personas con movilidad reducida en silla de ruedas convencionales. Para complementar el mismo, se realizó inicialmente un diseño conceptual para seleccionar la variante de diseño más adecuada, a partir de esto se realizó el diseño de las transmisiones que garantizan el avance y giro del dispositivo, posteriormente se realizaron los análisis de resistencia de los elementos estructurales mediante el método de los elementos finitos (MEF) y la selección de cojinetes de rodamiento, con ayuda del software SolidWorks ${ }^{\circledR}$ 2018, además se calcularon los costos de fabricación de un prototipo. Finalmente se construyó el prototipo, el cual fue sometido a pruebas durante su operación. Los principales resultados correspondientes al diseño conceptual, determinan a la variante tres como la más adecuada, variante que comprende una transmisión por cadenas para el accionamiento de la rueda motriz y una transmisión por engranajes de dientes rectos para garantizar los giros. El análisis de la efectividad económica mostró que la introducción del prototipo representó un ahorro cercano al $28 \%$ con respecto a la adquisición de dispositivos de esta similitud.
\end{abstract}

Palabras claves — Inclusión social, motor, sistema, transmisión de potencia.

\section{Device For Autonomous Mobility Of Conventional Electric WHEELCHAIRS USERS}

\footnotetext{
Abstract - Social inclusion for people with disabilities influences their personal development within the society in which they find themselves, variants and priority aids are available to allow this population in a situation of disability to function autonomously and without complications. the activities day to day programs. Taking into account this problem, this work aims to develop a device for the autonomous transport of people with reduced mobility in conventional wheelchairs. To complement this, a conceptual design was initially carried out to select the most appropriate design variant. From this, the design of the transmissions that
}

\% Dirección para correspondencia: arturocarmontesro@gmail.com

DOI: https://doi.org/10.24050/19099762.n25.2019.1350 
guarantee the advancement and rotation of the motor system was carried out, after which the resistance analysis of the structural elements by means of the finite element method (MEF) and the selection of rolling bearings, with the help of SolidWorks ${ }^{\circledR} 2018$ software, the manufacturing costs of a prototype were also calculated. Finally, the motor system was built, which was subjected to tests during its operation. The main results corresponding to the conceptual design, determine the variant three as the most appropriate, variant comprising a transmission by chains for the drive of the drive wheel and a gear transmission of straight teeth to ensure turns. The analysis of the economic effectiveness showed that the introduction of the prototype represented a saving close to $28 \%$ with respect to the acquisition of devices of this similarity.

Keywords-Social inclusion, motor, system, power transmission.

\section{DisPOSITIVO PARA MOBILIDADE AUTÔNOMA DE USUÁRIOS CONVENCIONAIS DE CADEIRAS DE RODAS ELÉTRICAS}

Resumo - Inclusão Social para pessoas com deficiência está influenciando seu desenvolvimento pessoal dentro da sociedade em que eles são encontrados, são variantes e auxílio de prioridade para habilitar esta população em situação de incapacidade, pode Navegar autonomamente e sem complicações em programas de atividades todos os dias. Para considerar este problema, o presente trabalho visa desenvolver um dispositivo para a circulação autônoma das pessoas com mobilidade reduzida em cadeiras de rodas convencionais. Para complementá-lo, um projeto conceitual foi inicialmente feito para selecionar o projeto mais adequado, baseado nesse layout de transmissões que garantam o avanço e a rotação do dispositivo, em seguida, foram a análise da resistência dos elementos estruturais usando o método de elementos finitos (MEF) e a seleção de pastilhas de rolamento, com a ajuda de SolidWorks ${ }^{\circledR}$ software de 2018, também foram calculados os custos de fabricação um protótipo. Finalmente, construiu o protótipo, que foi testado durante a operação. Os principais resultados para o projeto conceitual, determinar a variante três como a variante mais adequada, que compreende uma cadeia de transmissão para conduzir a roda motriz e transmissão de engrenagens de dente reto para Fixe as torções. A análise da eficácia econômica mostrou que a introdução do protótipo representou uma economia de cerca de $28 \%$ em relação a aquisição de dispositivos desta semelhança.

Palavras-chave-incluindo sistema de motor, social, transmissão de energia.

\section{INTRODUCCIÓN}

$\mathrm{E}^{\mathrm{n}}$ la actualidad el gobierno ecuatoriano ha prestado gran atención a las personas con discapacidad física [1], ya que esta puede limitar el desarrollo de dichas personas dentro de la sociedad [2]. Todos los ciudadanos cuentan con los mismos derechos y oportunidades en las distintas dimensiones sociales $\mathrm{y}$, por esto, la inclusión social ha logrado grandes cambios principalmente en la forma de pensar de la población [3], haciendo hincapié en la integración, la equidad, la cohesión, la justicia social, entre otros [4]. La inclusión social consiste en materializar la posibilidad de participación igualitaria de personas de una sociedad y su objetivo principal es asegurar que todas las personas tengan las oportunidades y los recursos necesarios para participar plenamente en la vida económica, social y política, para poder disfrutar una vida digna.

En función del grado de discapacidad de la persona se han desarrollado diferentes dispositivos que de alguna forma le faciliten la convivencia social [5]. Las sillas de ruedas son medios de transporte conducidos por otra persona o autopropulsados. Los autopropulsados tienen la posibilidad de ser auto guiado por parte del usuario, mediante un control accionado manualmente (joystick) o por medio de una interfase [6]. Las interfases permiten la comunicación del usuario con el equipo, ya sea reconociendo voces de mando o identificando gestos faciales, según el tipo de discapacidad que presenten [7], [8]. Para mejorar la funcionalidad de las sillas de ruedas convencionales [9], [10], [11], se han desarrollado variantes constructivas que pueden ser auto guiadas por un tercero o por el propio usuario. La variante "Power Pack Plus" es un sistema que integra un motor adaptable para una gran cantidad de tipos de silla de ruedas convencional [11], puede ser conducido de manera autónoma por el usuario, aunque en todos los casos se prevé la ayuda de un tercero. El "Wheeldrive" es una rueda que disponen en su centro de un motor incorporado, se elimina el joystick como interfaz para accionar los propulsores y tiene un panel para controlar las velocidades de marcha y estado de la batería [12]. Por su parte, el "Handbike" es un dispositivo que permite acoplarse a la silla de manera rápida, protege los pies y permite que el usuario controle la dirección y el movimiento, ya sea accionándolo manualmente o con la ayuda del motor eléctrico [13].

Cuanto mejor sean las características de desempeño de las sillas de ruedas, los precios de adquisición incrementan, a lo cual se suma que dichas variantes tecnologías no se producen o se comercializan a nivel nacional. La política de ayuda a las personas con discapacidad en sus extremidades está limitada a la donación de sillas ruedas convencionales, independientemente del grado de discapacidad y condición económica del usuario. Es por ello 
que se decide realizar el presente trabajo que tiene como objetivo desarrollar un dispositivo para el traslado autónomo de personas con movilidad reducida en silla de ruedas convencionales.

\section{Materiales y Métodos}

El trabajo se realizó en el período comprendido de marzo a septiembre del 2018, en el área de talleres de elaboración mecánica de la Universidad Técnica de Manabí.

\section{Materiales}

Software: SolidWorks ${ }^{\circledR} 2018$, (Student Edition); sistema operativo Windows ${ }^{\circledR} 10$.

Hardware: Acero SAE 1020; rodamientos de bolas de contacto angular, simple espacio, norma DIN 625-1, (6201-6202); tubo cuadrado SAE 1020 de $20 \times 20 \times 2$ $\mathrm{mm}$, rueda neumática "2,50 - 4", pernos SAE Grado 1, de cabeza hexagonal M6 x 1,0 x 50 - 18WS; cadena número 25; motor de 24 voltios de corriente continua modelo MY1016, motor "paso a paso" modelo C6244-9212K; joysticks análogo modelo YL-K1+M10, silla de ruedas "Roscoe K2-lite", catarinas, piñón libre, baterías de gel (24 V, 14 A/h), electrodos E-6011, (230-S) 1/8.

\section{Métodos}

Metodología para el diseño conceptual del dispositivo

Se procede a aplicar la metodología de despliegue de la función de calidad [14], [15] o (QFD). Para esto, se consideran las características que las personas con discapacidad sugieren que el producto contenga, sin dejar a un lado a las características de los productos ya existentes; además, se determinan las posibles variantes por medio de la relación entre las funciones y características que debe de cumplir el prototipo.

Metodología para el cálculo de la transmisión de potencia y velocidad a la rueda motora

A partir del establecimiento de la ecuación del momento de impulsión [16], se puede determinar inicialmente la potencia de diseño que se requerirá para el movimiento de la rueda motora, a partir de la base de que el torque necesario $(\tau)$ para hacerla girar es igual al momento de impulsión $\left(M_{\text {imp }}\right)$.

$$
\tau=M_{\text {imp }}=\frac{P \times 716}{n_{\text {mot }}}
$$

Donde: $\tau$, torque, N. m;

$P$, potencia de diseño, $h p$;

$n_{\text {mot }}$, revoluciones del motor, rpm.
Para la selección de las revoluciones $\left(n_{1}\right)$ del motor se realiza el cálculo de la velocidad de rotación de la rueda motora, consideración la velocidad de avance óptima de la silla, con un radio $(r)$ conocido, entonces la velocidad de rotación $\left(n_{m o t}=n_{2}\right)$ se determinará, como:

$$
v=\frac{r \pi n_{m o t}}{30} ; n_{m o t}=\frac{30 v}{\pi r}
$$

Donde: $v$, velocidad avance real, $\frac{m}{s}$;

$r$, radio de la rueda, $m$;

$n_{\text {mot }}$, revoluciones por minuto, rpm.

Si se conoce la velocidad $n_{\text {mot }}$ de rotación, entonces se sustituye (2) en (1), donde se puede determinar la potencia requerida para el accionamiento de la rueda o potencia de diseño, como:

$$
\text { Pdis }=P=\frac{M_{i m p} n_{m o t}}{716}
$$

El resto de los parámetros de diseño de la transmisión corresponde a una transmisión de potencia por cadena [17], donde se calculan la potencia del motor, relación de transmisión, tipo de cadena, número de dientes de la catarina y piñón, velocidad de salida de la transmisión, diámetros de la catarina y piñón, distancia entre centros y ángulo de contacto.

Metodología para el cálculo de la transmisión de potencia para el giro de la rueda motora

Para determinar la fuerza de resistencia al giro [16], se consideran dos movimientos: de avance de transferencia desde el centro del eje trasero con una velocidad $(v)$ en dirección del eje longitudinal y de giro del bastidor por el mismo punto indicado anteriormente, se tiene una velocidad angular $w_{g}=v / R$.

$$
Z_{g}=\frac{M_{r e s}}{L \cos \alpha}
$$

Donde:

$Z_{g}$, fuerza de resistencia al giro, $N$;

$M_{\text {res }}$, momento de resistencia, $\mathrm{Nm}$;

l, batalla, $m$;

$\alpha$, ángulo máximo de desviación, ${ }^{\circ}$; tiene:

Al despejar el momento de resistencia al giro de (4), se

$$
M_{r e s}=Z_{g}(L \cos \alpha)
$$

Esta variable se convierte en el momento o torque a vencer para poder realizar el giro, al considerar el ángulo 
máximo de desviación con un valor de $\left(45^{\circ}\right)$. Para esta potencia de diseño es necesario tomar como referencia (3), el momento involucrado es necesario para realizar el giro de la rueda motora, determinándose la potencia de diseño, como:

$$
\text { Pdis }=P=\frac{M_{\text {res }} n_{\text {mot }}}{716}
$$

Para esta acción se recurre a una transmisión de potencia por engranajes de dientes rectos, en una disposición de tipo engranaje planetario, según las variantes más adecuadas obtenidas en el diseño conceptual. A partir de este punto se aplica la metodología de diseño de engranajes de dientes rectos [17] y se consideran los siguiente parámetros de diseño: la potencia de la fuente energética, número de dientes, velocidad de salida real, diámetros de paso, distancia entre centros, velocidad de la línea de paso, carga transmitida, ancho de cara, calidad del piñón y engranaje, factor de flexión y picadura, distribución de carga, de tamaño, de espesor de borde, de servicio, relación de durezas, materiales para el piñón y engranaje.

Metodología para el cálculo de la resistencia de los elementos del dispositivo

El análisis de resistencia se realizó mediante el método de los elementos finitos en el software SolidWorks ${ }^{\circledR} 2018$. El método de análisis por elementos finitos se ha descrito ampliamente por varios autores [18], [19], [20]. Los pasos que comprenden esta metodología son: creación del modelo virtual (3D), definición de cargas y condiciones de frontera, asignación de materiales, mallado, simulación virtual y posprocesamiento de resultados.

Metodología para la selección de los cojinetes de rodamientos

Las magnitudes de las reacciones generadas en las zonas de apoyo de los árboles en un ambiente virtual de trabajo y la calculadora de rodamientos del software SolidWorks® 2018 [21], permitió hacer la selección de los cojinetes de rodamientos, los cuales fueron seleccionados bajo los estándares establecidos por la norma DIN 625-1.

\section{Metodología para la selección de elementos de sujeción}

La selección debe tener como referencia la tensión que recae en el elemento de sujeción [17], la cual es directamente proporcional al coeficiente entre la fuerza y el área de la sección.

\section{Metodología para evaluar el funcionamiento del dispositivo}

En esta etapa se llevaron a cabo pruebas de accionamiento, autonomía, dirección, inercia y pruebas con los usuarios. La prueba de accionamiento tenía como objetivo observar si se mantenía la velocidad de avance constante para tres tipos de superficies (asfalto, concreto, tierra) y pendientes (cero, inclinadas menores a $35 \%$, tanto en ascenso como descenso), así como determinar el rango de velocidades de avance de la silla. Se trabajó con el peso máximo que soporta la silla de ruedas convencional $(113,64 \mathrm{~kg})$.

Para evaluar la autonomía se determinó la distancia que el dispositivo podía desplazarse con el máximo peso a la velocidad promedio $(\approx 7 \mathrm{~km} / \mathrm{h})$. La fuente energética se encontraba a máxima carga. Por otra parte, para la dirección, se determinó el radio de giro de la silla de ruedas para ángulos de giro $\alpha \leq 45$, así como estabilidad de la misma tanto en giro como en vía sin obstáculos y, para la inercia, se determinó el desplazamiento total al suspender el paso de energía eléctrica al dispositivo, cuando este se encuentra a su máxima velocidad de avance.

Finalmente, en la prueba con los usuarios se evaluó el funcionamiento del dispositivo con personas de movilidad reducida en extremidades inferiores, los cuales fueron alumnos de la UTM y fueron seleccionados con base en los registros del departamento de inclusión social. Se aplicó una encuesta para conocer la satisfacción del usuario al utilizar el dispositivo.

\section{Análisis económico del desarrollo del prototipo}

Se elaboró una ficha de costos, en la cual se incluyeron los costos directos e indirectos, un margen de utilidad sobre la base autorizada y su respectivo impuesto al valor agregado. Se calculó la efectividad económica, como:

$$
E C=P D S-(P P)
$$

Donde:

$P D S$, es el precio actual de dispositivos similares, incluye envío e instalación, USD;

PP, precio del prototipo desarrollado, USD.

\section{Resultados y Discusión}

El desarrollo del diseño conceptual se realizó en función de tres aspectos, tales como: el aspecto económico, donde se considera el costo de producción, mantenimiento y reparación; el aspecto funcional que comprende, instalación, autonomía, operación, velocidad y rodadura y, finalmente, el aspecto de seguridad. Dentro de cinco variantes posibles de diseño (Tabla 2), la indicada con flechas de color azul (Tabla 1), tiene una ponderación de 31,6 \%. Al ser el valor más elevado entre las variantes (Tabla 3), se seleccionó como la opción más factible para el diseño del prototipo por cumplir con el mayor número de beneficios o soluciones perseguidas. 
Tabla 1 Relación de funciones y características

\begin{tabular}{|c|c|c|c|c|}
\hline N. ${ }^{\circ}$ & Funciones & & Características & \\
\hline & Opciones & 1 & 2 & 3 \\
\hline 1 & Fuente energética del sistema para marcha & Motor DC & Motor CI & \\
\hline 2 & Alimentación de la fuente energética & Electrica continua & $\begin{array}{l}\text { Combustibles } \\
\text { carburantes }\end{array}$ & \\
\hline 3 & N. ${ }^{\circ}$ de motores para marcha & Uno & Dos & \\
\hline 4 & Tipo transmisión de potencia para la marcha & Por banda & Por piñón & Por cadena \\
\hline 5 & $\begin{array}{l}\text { Tipo de piñón que transmite el torque a la rueda } \\
\text { motriz }\end{array}$ & Por piñón fijo & Por piñón libre & \\
\hline 6 & Fuente energética del sistema de giro & Motor DC & Actuador hidráulico & Actuador neumático \\
\hline 7 & Accionamiento del sistema de giro & Motor paso a paso & Actuadores eléctricos & $\begin{array}{l}\text { Bomba hidráulica o } \\
\text { compresor }\end{array}$ \\
\hline 8 & N. ${ }^{o}$ de motores para giro & Uno & Dos & \\
\hline 9 & Tipo de transmisión para el giro & Sin fin-corona & Engranajes planetarios & Por cadena o terminales \\
\hline 10 & Mando & Joystick & Por voz & \\
\hline 11 & Número de ruedas motoras & Una & Dos & \\
\hline 12 & Tipo de rueda motora & Maciza & Neumática & Inserto sólido \\
\hline 13 & Material de la estructura & Acero & Aluminio & \\
\hline
\end{tabular}

Tabla 2 Posibles variantes para el diseño conceptual

\begin{tabular}{cl}
\hline Variante \\
\hline 1 & $1.1-2.1-3.2-4.1-5.1-6.1-7.2-8.1-9.3-10.2-11.1-12.1-13.2$ \\
2 & $1.2-2.2-3.1-4.2-5.2-6.2-7.3-8.2-9.3-10.1-11.2-12.3-13.1$ \\
3 & $\mathbf{1 . 1}-\mathbf{2 . 1}-\mathbf{3 . 1}-\mathbf{4 . 3}-\mathbf{5 . 2}-\mathbf{6 . 1}-\mathbf{7 . 1}-\mathbf{8 . 1}-\mathbf{9 . 2}-\mathbf{1 0 . 2}-\mathbf{1 1 . 1}-\mathbf{1 2 . 2}-\mathbf{1 3 . 1}$ \\
4 & $1.1-2.1-3.2-4.3-5.1-6.1-7.1-8.1-9.2-10.2-11.2-12.2-13.2$ \\
5 & $1.1-2.1-3.1-4.1-5.1-6.1-7.2-8.2-9.3-10.1-11.2-12.1-13.2$ \\
\hline
\end{tabular}

Tabla 3. Evaluación de las variantes por medio de criterios

\begin{tabular}{|c|c|c|c|c|c|c|c|c|}
\hline Variante & $\begin{array}{c}\text { Cumple } \\
\text { tarea } \\
\text { global }\end{array}$ & $\begin{array}{l}\text { Realizable } \\
\text { en un } \\
\text { principio }\end{array}$ & $\begin{array}{c}\text { Dentro } \\
\text { de costos } \\
\text { permisibles }\end{array}$ & $\begin{array}{l}\text { Segura } \\
\text { para el } \\
\text { usuario }\end{array}$ & $\begin{array}{c}\text { Espacio } \\
\text { y fácil } \\
\text { instalación }\end{array}$ & $\begin{array}{l}\text { Preferida por } \\
\text { diseñador }\end{array}$ & No $(+)$ & $\begin{array}{l}\text { Valor, } \\
\%\end{array}$ \\
\hline 1 & + & + & 1 & + & - & - & 3 & 15,7 \\
\hline 2 & + & I & - & + & - & - & 2 & 10,5 \\
\hline 3 & + & + & + & + & + & + & 6 & 31,6 \\
\hline 4 & + & + & I & + & - & + & 4 & 21,1 \\
\hline \multirow[t]{2}{*}{5} & + & + & + & + & - & - & 4 & 21,1 \\
\hline & & & & & & & 19 & 100 \\
\hline
\end{tabular}

Cada una de las variantes se evaluó, con el propósito de evidenciar cuál era la más factible, sin que esta se saliera del marco de los aspectos definidos previamente. Se aplicó el siguiente criterio de evaluación: (+) Solución perseguida; (-) Eliminación de la solución; (/) Falta de información, recolectar información y re-evaluar.

El optar por el Despliegue de la función de calidad como metodología de diseño permitió discernir las funciones y características más relevantes que el dispositivo debería de cumplir, para satisfacer las necesidades de los usuarios de las sillas de ruedas convencionales. En este caso, el prototipo se asemejó a las sillas de ruedas eléctricas, con una variante más accesible que integra aspectos económicos, de funcionabilidad y seguridad.

\section{Descripción y principio de funcionamiento del dispositivo}

El dispositivo puede adaptarse a sillas convencionales, en general, mediante el posicionamiento de matrices de acople (Fig. 1) que se fijan en la parte inferior del asiento de la silla de ruedas. Cuenta con un sistema de dirección que permite el giro desde $0^{\circ}$ a $360^{\circ}$ en condiciones estáticas, 
con el accionamiento de un motor "paso a paso" que permite acceder a giros de grados más exactos. Igualmente, se pueden realizar giros en marcha sin necesidad de hacer paradas, donde los ángulos deben de ser $\alpha \leq 45^{\circ}$.

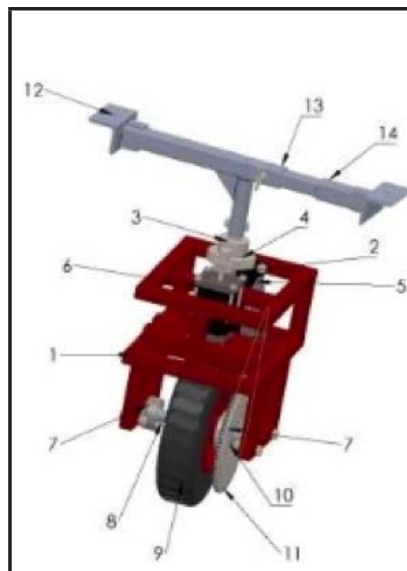

\begin{tabular}{|c|c|}
\hline ÍTEMS & $\begin{array}{c}\text { NOMBRE DE } \\
\text { COMPONENTES }\end{array}$ \\
\hline 1 & CHASÍS \\
\hline 2 & EJE MACIZO \\
\hline 3 & ENGRANAJE \\
\hline 4 & RODAMIENTO DE GIRO \\
\hline 5 & MOTOR DE MARCHA \\
\hline 6 & MOTOR DE GIRO \\
\hline 7 & CHUMACERA \\
\hline 8 & ÁRBOL CON ROSCA \\
\hline 9 & RUEDA \\
\hline 10 & PIŃÓN LIBRE \\
\hline 11 & CATARINA \\
\hline 12 & MATRIZ DE ACOPLE \\
\hline 13 & ACOPLE HEMBRA \\
\hline 14 & ACOPLE MACHO \\
\hline
\end{tabular}

Fig. 1. Diseño virtual del dispositivo con sus partes

Tabla 4. Características del diseño del dispositivo y la silla de ruedas "Roscoe K2-lite"

\begin{tabular}{lcc}
\hline Variables medidas & Unidad & Valor \\
\hline Masa total (silla + dispositivo + baterías) & $\mathrm{kg}$ & 36,81 \\
Peso del dispositivo & $\mathrm{kg}$ & 9,77 \\
Máxima capacidad de peso de la silla (MCP) & $\mathrm{kg}$ & 113,64 \\
Peso transferido a la rueda motora con el MCP & $\mathrm{kg}$ & 59,55 \\
Batalla & $\mathrm{m}$ & 0,45 \\
Profundidad de asiento & $\mathrm{mm}$ & 406,4 \\
$\begin{array}{l}\text { Distancia del suelo al asiento de la silla } \\
\text { Peso transferido a la rueda en una pendiente } \\
\text { del 4 \%, con la masa total }\end{array}$ & $\mathrm{mm}$ & 457,2 \\
\hline
\end{tabular}

El sistema se alimenta con baterías de gel (12 V y 14 $\mathrm{A} / \mathrm{h}$ ) situadas en la parte trasera de la silla de ruedas y es capaz de alcanzar una velocidad máxima de $14 \mathrm{~km} / \mathrm{h}$, con una autonomía o desplazamiento de $28 \mathrm{~km}$ con una sola carga. Un piñón libre en el árbol que recibe la potencia del motor, permite el rodaje libre en una pendiente sin forzar el motor o gastar energía, además de permitir su impulso manual sin esfuerzo, en caso que este se encuentre sin energía para su respectivo funcionamiento. A continuación, se muestran las características del dispositivo y la silla de ruedas (Tabla 4), tomando en cuenta que el dispositivo puede integrarse a sillas de ruedas convencionales que tengan una altura del piso al asiento mayor a 457,2 mm o su equivalente a 18 ".

Resultados de los cálculos de la transmisión de potencia y velocidad a la rueda motora

La potencia de diseño es una de las variantes más relevantes dentro de los cálculos; sin embargo, en muchos problemas descritos en libros, dicho parámetro está establecido por el autor, no se incluye o se descarta dicho cálculo. El diseño de la transmisión permitió obtener una reducción de la velocidad de rotación cercana a $344 \mathrm{rpm}$, con una velocidad de avance de la silla de ruedas alrededor de $14 \mathrm{~km} / \mathrm{h}$, la cual es similar a las velocidades de operación empleadas (6 a $15 \mathrm{~km} / \mathrm{h}$ ) en las sillas de ruedas eléctricas en el mercado internacional [22], [12], [23].

Las transmisiones de potencia por cadena se emplean cuando hay limitaciones de espacio (como es el caso de este análisis), permite una gran maniobrabilidad en espacios reducidos y posibilita ciertas velocidades (bajas y medias) [24]. Los resultados de los cálculos de la transmisión de potencia por cadena se muestran en la (Tabla 5), donde la rueda motora requiere un torque de $0,2808 \mathrm{~kg} \cdot \mathrm{m}$, según las condiciones de la superficie de rodamiento y de operación de la silla. Para las condiciones de la silla de ruedas objeto de estudio se escogió una rueda con una denominación de "2,50-4" la cual soporta una presión de inflado de $241 \mathrm{kPa}$ (35 psi) y un diámetro exterior de $210 \mathrm{~mm}$.

Tabla 5. Visualización de resultados para el diseño de transmisión por cadena

\begin{aligned} & Aplicación: Tracción del dispositivo \\ & Fuente/tipo: Motor eléctrico-transmisión mecánica \\ & Máquina movida: Silla de ruedas convencional \\ & Entrada de potencia: $0,3353 \mathrm{hp} \approx 250 \mathrm{~W} \\ &$ Factor de servicio: 1,00 \\ & Velocidad de entrada: $2500 \mathrm{rpm} \\ &$\hline Datos calculados: \\ & \hline Velocidad de salida deseada: $344 \mathrm{rpm} \\ &$\hline Potencia de diseño: $0,1349 \mathrm{hp} \approx 100,59 \mathrm{~W} \\ &$\hline\end{aligned}




\begin{tabular}{|c|c|c|c|c|c|}
\hline \multicolumn{6}{|l|}{ Decisiones de diseño - Tipo de cadena y números de dientes } \\
\hline Cantidad de hileras: & 1 & 1 & 2 & 3 & 4 \\
\hline Factor por hileras: & 1,0 & & & & \\
\hline Potencia requerida por hilera: & $0,1349 h p$ & 1,0 & 1,7 & 2,3 & 3,3 \\
\hline Número de cadena: & 25 & & & & \\
\hline Paso de la cadena: & 0,25 pulg & & & & \\
\hline Número de dientes-piñón motriz: & 11 & & & & \\
\hline Número de dientes-catarina: & 79,9419 & & & & \\
\hline Número de dientes elegido: & 80 & & & & \\
\hline \multicolumn{6}{|l|}{ Datos calculados: } \\
\hline Velocidad real de salida: & $343,75 \mathrm{rpm}$ & & & & \\
\hline Diámetro de paso - catarina motriz: & 0,8874 pulg & & & & \\
\hline Diámetro de paso - catarina conducida: & 6,3678 pulg & & & & \\
\hline \multicolumn{6}{|l|}{ Distancia entre centros, longitud de cadena y ángulo de contacto } \\
\hline Distancia nominal entre centros: & 40 & \multicolumn{4}{|c|}{ Rec. (30-50 pasos) } \\
\hline Longitud de la cadena calculada: & 128,5149 pasos & & & & \\
\hline Número de pasos específicos: & 128 pasos & \multicolumn{4}{|c|}{ Rec. Número par. } \\
\hline Longitud real de la cadena: & 32 pulg & & & & \\
\hline Distancia calculada real entre centros & 42,6634 pasos & & & & \\
\hline Distancia real entre centros: & 10,6659 pulg & \multirow{2}{*}{\multicolumn{4}{|c|}{ Mayor a $120^{\circ}$}} \\
\hline Ángulo de contacto - catarina motriz: & $147^{\circ}$ & & & & \\
\hline Ángulo de contacto - catarina conducida: & $213^{\circ}$ & & & & \\
\hline
\end{tabular}

Tabla 6. Resultados para el diseño de transmisión por engranajes rectos

\begin{tabular}{|c|c|c|c|c|}
\hline \multicolumn{5}{|c|}{ DISEÑO DE ENGRANAJES RECTOS } \\
\hline \multicolumn{5}{|l|}{ Datos iniciales: } \\
\hline & Potencia de entrada: & \multicolumn{3}{|l|}{$P=50 \mathrm{~W} \approx 0,0671 \mathrm{hp}$} \\
\hline & Velocidad de entrada: & \multicolumn{2}{|l|}{$n_{p}=8 \mathrm{rpm}$} & \\
\hline & Paso diametral: & \multicolumn{2}{|l|}{$P_{d}^{p}=16$} & \\
\hline & Número de dientes del piñón: & \multicolumn{2}{|l|}{$N_{p}^{a}=15$} & \\
\hline & Velocidad de salida deseada: & \multicolumn{2}{|l|}{$n_{G}=3 \mathrm{rpm}$} & \\
\hline & Número calculado de dientes de engranaje: & \multicolumn{2}{|l|}{40} & \\
\hline & Número de dientes escogidos para el engranaje: & \multicolumn{2}{|l|}{$N_{G}=40$} & \\
\hline \multicolumn{5}{|c|}{ Datos calculados: } \\
\hline & Velocidad real de salida: & \multicolumn{2}{|l|}{$n_{G}=3 \mathrm{rpm}$} & \\
\hline & Relación de engranaje: & \multicolumn{2}{|l|}{$m_{G}=2,667$} & \\
\hline & Diámetro de paso, piñón conductor: & \multicolumn{2}{|l|}{$D_{P}=0,9375$ pulg } & \\
\hline & Diámetro de paso, piñón conducido: & \multicolumn{2}{|l|}{$D_{G}=2,500$ pulg } & \\
\hline & Distancia entre centro: & \multicolumn{2}{|l|}{$C=1,7188$ pulg } & \\
\hline & Velocidad de la línea de paso: & \multicolumn{2}{|l|}{$v_{l}=1,9635 \mathrm{pies} / \mathrm{min}$} & \\
\hline & Carga transmitida: & \multicolumn{2}{|l|}{$W_{l}=1127,7311 \mathrm{lb}$} & \\
\hline \multicolumn{5}{|c|}{ Ingreso de datos secundarios: } \\
\hline & & Min. & Norm. & Máx \\
\hline & Lineamientos para ancho de cara (pulg): & 0,50 & 0,75 & 1,00 \\
\hline & Ancho de cara: & \multicolumn{2}{|l|}{$F=0,75$} & \\
\hline & Relación: Ancho de cara/diámetro del piñón: & & \\
\hline & Intervalo recomendado de la relación: & \multicolumn{2}{|l|}{$F / D_{P}<2,00$} & \\
\hline & Coeficiente elástico seleccionado: & \multicolumn{2}{|l|}{$C_{p}=2300$} & \\
\hline & Número de calidad seleccionado: & \multicolumn{2}{|l|}{$Q_{v}=5$} & \\
\hline & Factor de geometría para flexión: & & \\
\hline & Piñón conductor: & \multicolumn{2}{|l|}{$J_{P}=0,25$} & \\
\hline & Piñón conducido: & \multicolumn{2}{|l|}{$J_{G}=0,37$} & \\
\hline & Factor de geometría para picadura: & \multicolumn{2}{|l|}{$I=0,094$} & \\
\hline
\end{tabular}


Resultados de los cálculos de la transmisión de potencia para giro

Las transmisiones por engranajes de dientes rectos [25] permiten transmitir grandes magnitudes de potencia, son considerablemente más económicas que otro tipo de engranajes, no presentan empuje axial y su montaje se torna relativamente sencillo al poder utilizar rodamientos de bolas. Los resultados de los parámetros de diseño de la transmisión de potencia por engranajes de dientes rectos, se detallan en la Tabla 6.

La metodología de este tipo de transmisión involucra el cálculo de la resistencia a los esfuerzos de flexión y de picadura para garantizar la disminución de los costos de mantenimiento, reparación y reposición, es decir, garantizar el incremento de la vida útil de los elementos de transmisión y la confiabilidad del dispositivo en general. Por tal motivo, se seleccionó un acero SAE 1020.

Resultados de resistencia mecánica del árbol, chasis y piñones del sistema

Tensiones, deformaciones y factor de seguridad del árbol

Los resultados obtenidos del software de SolidWorks ${ }^{\circledR}$ 2018 determinaron que las tensiones máximas de von Mises alcanzan valores de 365,2 kPa, (Fig. 2), los cuales se registran en la zona más cercana donde se aplica el torque. La deformación alcanzó un valor máximo de $0,0000133 \mathrm{~mm}$ en la zona de tensión máxima, lo cual no afectó la rigidez del árbol, dado que son inferiores al 10 $\%$ de su longitud. Con respecto al factor de seguridad, se destaca que es capaz de resistir las condiciones de operación empleadas en el cálculo, pues toma valores mínimos de 1,7 .

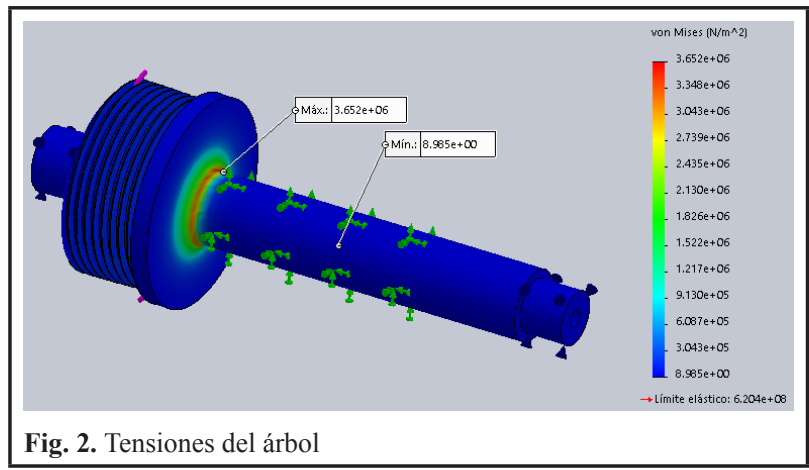

Resultados de la resistencia del sistema de dirección

Tensiones, deformaciones y factores de seguridad

El piñón y el engranaje fueron diseñados bajo las normas de "AGMA, Norma 917-B97" las variables involucradas en el diseño se encuentran en la Tabla 6. Para el piñón, las máximas tensiones de von Mises estuvieron alrededor de los $5529 \mathrm{kPa}$, (Fig. 3). La zona más cargada fue la sección del acoplamiento con el eje del motor de paso, mientras que las tensiones generadas en los dientes tienen valores cercanos a $1670 \mathrm{kPa}$. Para el engranaje, las tensiones de von Mises alcanzan valores de $1300 \mathrm{kPa}$, en los dientes, los valores son cercanos a $171,7 \mathrm{kPa}$. Las deformaciones para el piñón tuvieron un valor máximo de $0,0002489 \mathrm{~mm}$ y para los dientes un valor $0,00006291 \mathrm{~mm}$, lo cual no afecta la rigidez de este elemento; las deformaciones para el engranaje fueron de $0,000003847 \mathrm{~mm}$. El análisis del factor de seguridad evidenció que ambos elementos tienen la capacidad de resistir los esfuerzos que se originan en las condiciones de operación estudiadas.

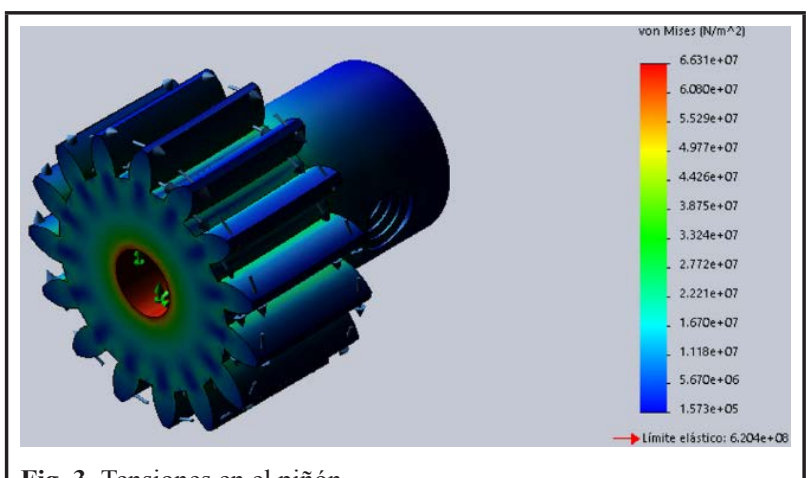

Fig. 3. Tensiones en el piñón

Resultados de resistencia de la estructura

\section{Tensiones y deformaciones}

El análisis de resistencia de la estructura mostró que las tensiones de von Mises máximas pueden alcanzar valores de $422 \mathrm{kPa}$, (Fig. 4.), las cuales no comprometen la integridad de la estructura del dispositivo. El desplazamiento máximo mostró una magnitud de $0,05898 \mathrm{~mm}$.

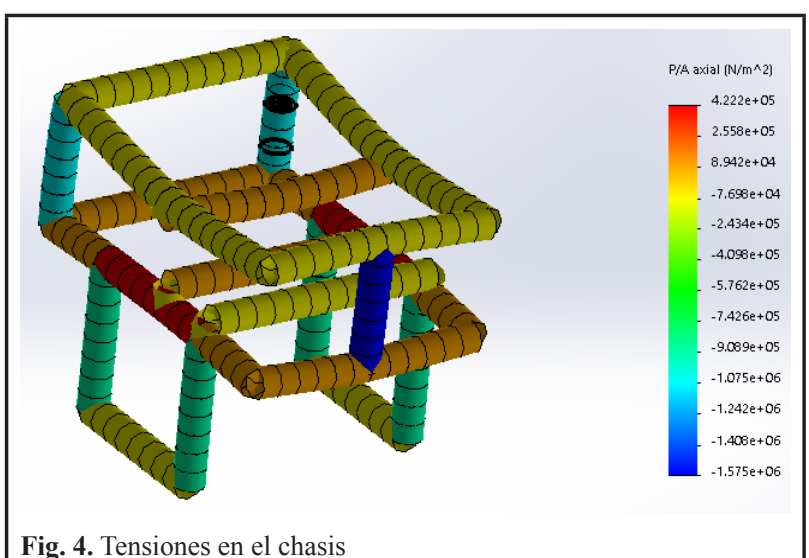




\section{Resultado de la selección de los rodamientos}

Para el árbol motriz se seleccionaron cojinetes de rodamiento del tipo (DIN 6201), pues el diámetro interior de este satisface las dimensiones de las secciones de apoyo del árbol, además, cumple con los requisitos de durabilidad con una vida en horas de $7,808 \cdot 10^{11}$. Para el sistema de dirección se seleccionó un cojinete de rodamiento (DIN 6202), pues cumple con las dimensiones requeridas y presenta una durabilidad de vida en horas de $1,908 \cdot 10^{22}$.

Resultado de la selección de tornillos o elementos apernados

Para la fijación del árbol se escogió una unión apernada para lo cual se seleccionaron pernos SAE Grado 1. Los resultados del análisis de los esfuerzos que actúan sobre la unión apernada se muestran en la (Tabla 7).

Tabla 7. Grados y tensiones cortantes

\begin{tabular}{cccc}
\hline $\begin{array}{c}\text { Cálculo para } \\
\text { el perno }\end{array}$ & $\begin{array}{c}\text { Fuerza } \\
F=27,5 \mathrm{lb}\end{array}$ & $\begin{array}{c}\text { Área } \\
A=0,049 \text { pulg}^{2}\end{array}$ & $\begin{array}{c}\text { Tensión cortante } \\
\tau=0,56122 \mathrm{Kpsi}\end{array}$ \\
\hline $\begin{array}{c}\text { Pernos } \\
\text { Acero de }\end{array}$ & $\begin{array}{c}\text { SAE Grado 1 } \\
(55-33 \mathrm{Kpsi}\end{array}$ & $\begin{array}{c}\text { Grado ASTM } \\
\text { (55-33) Kpsi }\end{array}$ & $\begin{array}{c}\text { Grado } 4.6 \\
\text { (225) Mpa }\end{array}$ \\
\hline
\end{tabular}

\section{Resultados de las uniones soldadas}

La acción de soldar la estructura o chasis, por la posición geométrica de las piezas a unir, corresponden a soldaduras a tope, que para este caso fueron soldaduras "A tope en T" y "A tope en L".

Existen algunas recomendaciones importantes cuando se realizan soldaduras a tope, entre ellas la más relevante es la de penetración completa y continua en toda la longitud [26]. Se optó por utilizar la soldadura por arco eléctrico con electrodo revestido, donde específicamente se usaron el electrodo E-6011 y E-7018. Este último electrodo se utilizó para realizar la unión del engranaje de dirección con el eje macizo que se une con el sistema de acople (Fig. 1).

\section{Resultados de la evaluación del funcionamiento del} dispositivo

Se comenzó por la elaboración del árbol de la rueda motora $\mathrm{y}$, posteriormente, se fabricó el chasis, elementos del sistema de dirección y las secciones de sujeción. Finalmente, se realizaron las uniones y acoples correspondientes y se obtuvo un prototipo con las dimensiones del diseño virtual (Fig. 5).

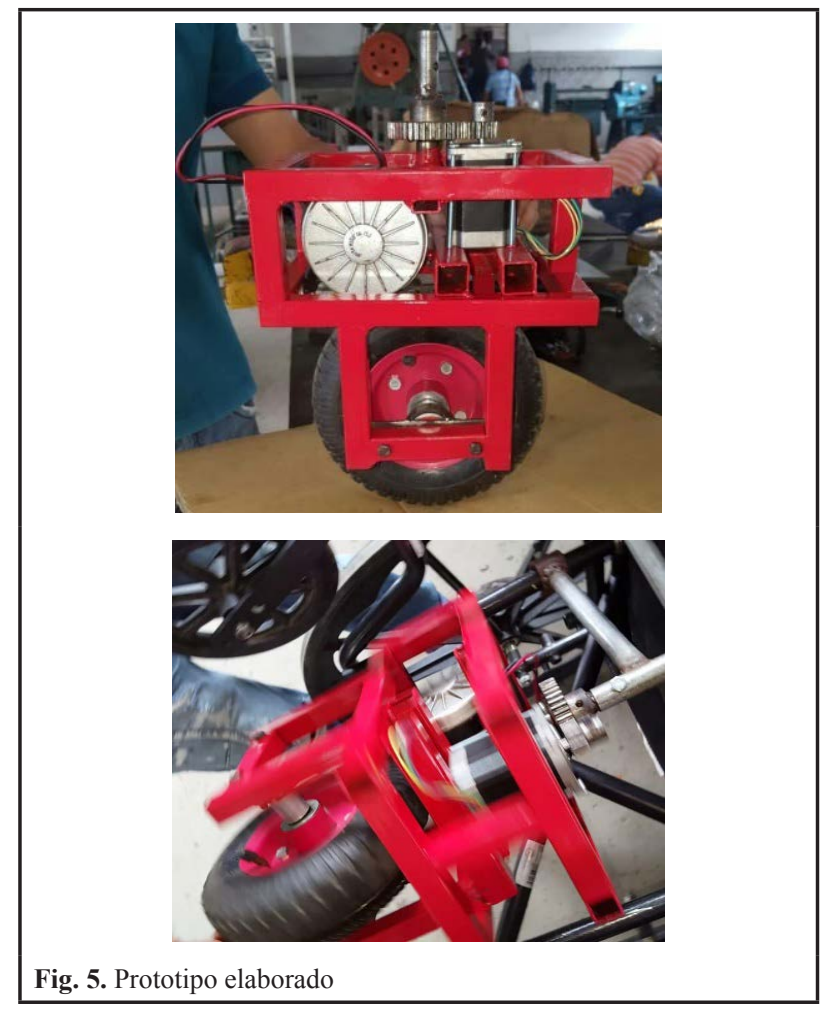

Las pruebas de funcionamiento realizadas mostraron que la transmisión por cadena, satisface la demanda de potencia durante el movimiento del dispositivo, pudiendo alcanzar la velocidad de avance establecida. El motor fue capaz de accionar el dispositivo en un régimen de carga máxima de la silla de ruedas, con un sistema de alimentación con $24 \mathrm{~V}$ y $14 \mathrm{~A} / \mathrm{h}$, (Tabla 8). De igual forma, se corroboró que el sistema de rodaje garantiza el tráfico en las distintas condiciones de superficies de rodadura, venciendo los obstáculos propios de las superficies. El motor y la transmisión garantizaron la potencia necesaria para vencer pendientes entre un (4 a 12) \%. El mecanismo de trinquete del piñón garantiza el movimiento sin accionamiento del motor durante los descensos. La autonomía o desplazamiento máximo del dispositivo fue de $28 \mathrm{~km}$. Al bloquear el paso de energía al dispositivo, cuando este se desplaza a su máxima velocidad de avance, se obtiene un desplazamiento por inercia cercano a los $3 \mathrm{~m}$.

Los resultados de la prueba de dirección evidenciaron que se pueden realizar giros en marcha sin que ocurran vuelcos o pérdidas de la misma, siempre y cuando el ángulo sea $\alpha \leq 45^{\circ}$. También se determinó que en parada se pueden generar giros mayores a $45^{\circ}$ hasta completar los $360^{\circ}$, lo cual permite realizar el retroceso de la silla de ruedas.

Las pruebas para determinar la satisfacción del usuario mostraron que, con respecto a la comodidad, maniobrabilidad, autonomía y desempeño del dispositivo desarrollado, hubo una satisfacción del $100 \%$. 
Tabla 8. Características del dispositivo desarrollado

\begin{tabular}{|c|c|c|c|}
\hline Características & $\begin{array}{l}\text { Silla de ruedas eléctrica por } \\
\text { mando (Joystick) }\end{array}$ & $\begin{array}{c}\text { Motor acoplable a sillas de ruedas } \\
\text { convencionales }\end{array}$ & Dispositivo desarrollado \\
\hline Capacidad de peso Admisible, $\mathrm{Kg}$ & 113 a 136 & 135 a 200 & 113,64 \\
\hline Número de baterías & \multicolumn{2}{|c|}{1 a 2} & 1 a 2 \\
\hline Voltaje, V & \multicolumn{2}{|c|}{12 o 24} & 12 o 24 \\
\hline Amperaje/hora, A/h & 17 a 26 & 16 a 22 & 14 \\
\hline Potencia de motor, $\mathrm{W}$ & 180 a 300 & 200 a 300 & 250 \\
\hline Motor, rpm & \multicolumn{2}{|c|}{100 a 10000} & 2650 \\
\hline Voltaje Motor, V & 24 & 24 a 12 & 24 a 12 \\
\hline Velocidad de avance, $\mathrm{Km} / \mathrm{h}$ & 5 a 30 & 5.5 a 13 & 0 a 14 \\
\hline Autonomía, Km & 16 a 20 & 13 a 15 & 28 \\
\hline Pendiente, $\%$ & 3 a 8 & 12 a 20 & 4 a 12 \\
\hline Tipo de rueda & Neumática & Neumática & Neumática \\
\hline Radio de giro, $\mathrm{m}$ & - & - & 0,62 \\
\hline Precio USD & 1700 a 5000 & 1200 a 7500 & 854 \\
\hline
\end{tabular}

Los precios y datos técnicos han sido tomado de una empresa en particular Ortowed. Sin embargo, muestran similitudes con otras empresas expendedoras de productos similares (Ortovitality, SCI Geratría, Merits, entre otros).

\section{Análisis económico de la fabricación}

Con el propósito de identificar los posibles gastos que tendría este tipo de desarrollo, se llevaron a cabo cálculos de gastos que se pueden generar durante la construcción del prototipo (Tabla 9). De esta manera el precio de la construcción toma un valor real con base en diferentes parámetros que están presentes en el proceso de la fabricación dentro de un taller dedicado a este oficio o afines a este.

\section{$E C=346 U S D$}

Según el valor calculado, se da un ahorro de más del $28 \%$ con respecto a un dispositivo similar "Power Pack Plus" que se puede comprar, con un valor de envío e instalación agregado, esto permite determinar que la construcción de un dispositivo para sillas de ruedas convencionales es una opción económica con respecto a la adquisición de equipos de esta índole. Al evaluar la efectividad económica del desarrollo del prototipo, se puede indicar que se consolida como una opción más accesible y económica para las personas que ya poseen una silla de rueda convencional [7], [8], [27].

Tabla 9. Ficha de gastos del dispositivo

\begin{tabular}{lcc}
\hline \multicolumn{3}{c}{ Fichas de precios y sus componentes } \\
\hline \multicolumn{3}{l}{ Empresa: Escuela de Ing. Mecánica } \\
\hline Plan de Producción: & 1 \\
\hline \multicolumn{2}{l}{ Producto: $\quad$ Dispositivo para silla de ruedas convencionales } \\
\hline Conceptos de gastos & Fila & Total unitario \\
\hline Materia prima y materiales & 1 & 368,30
\end{tabular}

\begin{tabular}{llc} 
Costos de manufactura & 2 & 130,00 \\
\hline Subtotal (Gastos de elaboración) & $\mathbf{3}$ & $\mathbf{4 9 8 , 3 0}$ \\
\hline Gastos de fuerza de trabajo & 4 & 100,00 \\
\hline Subtotal (Gastos directos 3+4) & $\mathbf{5}$ & $\mathbf{5 9 8 , 3 0}$ \\
\hline $\begin{array}{l}\text { Gastos indirectos de producción (10 \% } \\
\text { de 5) }\end{array}$ & 6 & 59,83 \\
Gastos de distribución y ventas (3 \% de 5) & 7 & 17,95 \\
Gastos bancarios (1\% de 5) & 8 & 5,98 \\
\hline Subtotal (Gastos Indirectos 5+6+7+8) & $\mathbf{9}$ & $\mathbf{6 8 2 , 0 6}$ \\
\hline $\begin{array}{l}\text { Margen de utilidad sobre la base autorizada } \\
\text { (15\% de 5) }\end{array}$ & 10 & 89,75 \\
Precio por unidad (9+10) & 11 & 771,81 \\
IVA (12 \%) & 12 & 81,85 \\
Precio por unidad incluido IVA & 13 & $\$ 853,66$ \\
\hline Total, USD (redondeo del valor) & $\mathbf{1 4}$ & $\mathbf{\$ 8 5 4}$ \\
\hline
\end{tabular}

\section{Conclusiones}

La mayoría de las personas con movilidad reducida cuentan con una silla de ruedas convencionales, cuando tienen que trasladarse grandes distancias necesitan de la ayuda de un familiar, no se adquiere una silla de ruedas eléctrica por la situación económica, por esta razón, se decide desarrollar un dispositivo que modifique a la silla de ruedas convencional y permita compararla con sillas de ruedas eléctrica, promoviendo un ahorro en precios y una igualdad en desempeño, permitiéndole una mayor autonomía al usuario al momento de ir a cumplir sus actividades diarias. 
El prototipo de dispositivo desarrollado permite la movilidad autónoma de una silla de ruedas convencional, conservando sus características y funcionalidad original. Se determinó que el diseño satisface las exigencias técnicas realizadas por el grupo de estudiantes para el funcionamiento de la silla de rueda en los distintos ambientes de trabajo.

El dispositivo desarrollado puede ser usado por personas que tengan movilidad en sus miembros superiores, pues dispone de una interfaz de Joystick que debe ser accionada para que se lleve a cabo el desplazamiento (avance, retroceso, giros). Sin embargo, el diseño permite que a futuro se pueda incluir una interfaz más avanzada (por comando de voz o expresiones faciales), lo cual ampliaría su uso a personas con movilidad reducida de los miembros superiores.

El diseño asistido por las técnicas CAD/CAE permitió la optimización del diseño, así como una adecuada selección de materiales, análisis de resistencia y simulación virtual del funcionamiento. Las pruebas de operación (avance y giros) realizadas al prototipo, permitieron determinar la estabilidad del dispositivo durante el funcionamiento, además de cumplir con los requisitos exigidos de autonomía (velocidad, potencia, operación, rodadura) y seguridad para el usuario.

La variante propuesta se puede comparar con sillas de ruedas existentes en el mercado, las cuales son similares desde el punto de vista de la funcionabilidad, seguridad, posibilidades de transporte y autonomía. El conjunto dispositivo y silla de ruedas convencional desarrollado posee precios inferiores a las sillas de ruedas que se comercializan actualmente en el mercado ecuatoriano; al compararse se obtiene un ahorro de más del $28 \%$ cercano a 350 USD, además, se denota que la implementación del dispositivo a nivel nacional presenta la ventaja de que elementos y materia primas se encuentran al alcance para poder construirlo y llevar a cabo los mantenimientos correspondientes.

\section{REFERENCIAS}

[1]. C. Valencia y M. E. Bernal. Institucionalidad y marco legislativo de la discapacidad en el Ecuador. Santiago CEPAL, 2016. Capítulo IV, 23-38.

[2]. Fioril P., del Rocío M., Cloquell Ballester V. A. Determinación antropométrica para mobiliario escolar destinado a niños con discapacidad motriz en Ecuador. Ciencia \& trabajo, 17, 154-158, 2015.

[3]. Cortés-Guerrero W. R., González M. D., Rodríguez Á. B. La inclusión de los estudiantes con discapacidades y/o necesidades educativas especiales desde la sociedad ecuatoriana. Maestro y Sociedad, 13, 424-435, 2016.
[4]. N. A. V. Mero, Impacto en la calidad de vida de las personas con discapacidad, posterior al plan de intervención del Bono "Joaquín Gallegos Lara", en Ecuador. Dominio de las Ciencias, 2, 189200, 2016.

[5]. Guajardo Ramos E. Red iberoamericana de expertos en la convención de los derechos de las personas con discapacidad. La Integración y la Inclusión de alumnos con discapacidad en América Latina y el Caribe (2017). Consultado el 20 de enero de 2019 en: http://repositoriocdpd.net:8080/handle/123456789/1979

[6]. Jazzy Electric Wheelchairs. Modelos de sillas de reudas (2017). Consultado el 20 de enero de 2019 en: https://www.jazzy-electricwheelchairs.com/

[7]. Olivares Carrillo C. Y. Diseño y construcción de una interfaz cerebro computadora para el control de una silla de ruedas como ayuda a personas con discapacidad motriz . Master thesis, Universidad del Norte, 2017.

[8]. Cifuentes A., Plaza M., Rondón S. Diseño de un módulo de transformación para sillas de ruedas eléctricas en silla de ruedas inteligente. Ciencia y poder aéreo, 11, 3, 2016.

[9]. Verdi C., Jesenia K. Diseño de acople mecatrónico para automatización de sillas de ruedas convencionales. Tesis de grado, Ingeniero Mecatrónico, PUCP, 2017.

[10]. Morales Perdomo A., Ribera Carné D., Rodríguez Mestres M. Motorización independiente de una silla de ruedas. Bachelor's thesis, Universidad Politécnica de Catalunya, 2015.

[11]. Ortopedia Torrejon. Estudio de la pisada (22 Mayo 2014). Consultado el 20 de enero de 2019 en: https://www. ortopediatorrejon.com/noticias.html\#top

[12]. Ortowed. Su calidad de vida, nuestro compromiso (16 Enero 2018). Consultado el 20 de enero de 2019 en: https://www. ortoweb.com/breezy-250-silla-de-ruedas-de-acero-plegableautopropulsable\#product-tabs

[13]. SCI Geratría. La boutique de la salud (8 Abril 2015). Consultado el 20 de enero de 2019 en: https://www.sci-geriatria. com/?s=handbike

[14]. Sánchez J. G. L., Grajales M. Á. J., Aguilar J. S. Despliegue de la Función de Calidad para el Diseño de Limpiador Automático de Rodillo. Conciencia Tecnológica, 51-54, 2016.

[15]. Escobar O., Sofía E., Rodríguez C., Julio C., Velasco D., Germán O. Despliegue de la funcion calidad (QFD): beneficios y limitaciones detectados en su aplicación al diseño de prótesis mioeléctrica de mano. Ingeniería e Investigación, 25, 30-38, 2005.

[16]. Chudakov D. A. Fundamentos de la teoría y el cálculo de tractores y automóviles. URSS: Mir Moscú, 1997. Capítulo 8, 246-252.

[17]. Moott R. L. Diseño de elementos de máquinas, Mexico: Pearson educación, 2006. Capítulo 7; 9, 18, 283-295, 366-430, 715-717.

[18]. Alves Filho A. Elementos Finitos-A base da tecnologia CAE. Editora Saraiva, 2018. Capítulo 1.

[19]. González-Estrada, Octavio A., Natarajan S., Graciano C. Reconstrucción de tensiones para el método de elementos finitos con mallas poligonales. Revista UIS Ingenierías, 16, 23-34, 2017.

[20]. Medina R., Salas M., Luco R., Bertram,V. Analisis de estructuras navales mediante el Método de Elementos Finitos. Sintesis Tecnológica, 27-36, 2017.

[21]. Núñez G., G. R., Polo M., Joshep S. Diseño de una máquina ordeñadora accionada por una fuerza no convencional, Tesis de Grado, Ingeniero Mecánico, USS, 2018. 
[22]. SCI Geratría. La boutique de la salud, (14 Diciembre 2016). Consultado el 20 de enero de 2019 en: https://www.sci-geriatria. $\mathrm{com} /$ ? $\mathrm{s}=$ wheeldrive

[23]. Ortovitality.Ortovitality, facilitamos tu vida (2018). Consultado el 20 de enero de 2019 en: http://ortovitality.com/home/61-argon-2. html

[24]. Ochoa, G. K., MESA P. A. G. Diseño y construcción de una instalación para el estudio de las transmisiones de potencia mecánica. Doctoral dissertation, Universidad Tecnológica de Pereira, Facultad de Tecnologías, Tecnología Mecánica, 2015.

[25]. Neyra Dueñas E. J. Análisis comparativo de métodos para el diseño de Engranajes Cilíndricos de Dientes Rectos. Tesis de grado, Ingeniero Mecánico Eléctrico, UJCM 2018.

[26]. Universidad de Castilla-La Mancha. Escuela de Ingenieros Agrónomos. Ingeniería Rural, (2016). Consultado el 20 de enero de 2019 en: https://previa.uclm.es/area/ing_rural/trans_const/ elementosconstruccion02.pdf

[27]. Higuera Ó., Espitia H., Méndez D. Prototipo de silla de ruedas comandada por voz empleando hmm en un ambiente controlado. Ingeniería Investigación y Desarrollo, 16, 26-38, 2016. 